\title{
Subacute Femoral Compressive Neuropathy from Iliacus Compartment Hematoma
}

\author{
Farhad Pirouzmand and Rajiv Midha
}

\begin{abstract}
Background: Traumatic retroperitoneal hematoma in the iliacus muscle is an unusual but potentially serious cause of femoral compression neuropathy. Case report: We describe the clinical, imaging, and management features of a case of traumatic iliacus retroperitoneal hematoma with delayed manifestation of femoral neuropathy. Discussion: The anatomical substrate for hematoma formation with subacute compression of the femoral nerve is emphasized. A subacute compartment syndrome with progressive edema, swelling and ischemia of iliacus compartment is suggested as the underlying cause. Early fasciotomy with or without hematoma evacuation should be considered in order to provide rapid decompression and to minimize the chance of permanent nerve injury.
\end{abstract}

RÉSUMÉ: Neuropathie compressive fémorale subaiguë due à un hématome de la loge iliaque. Introduction: L'hématome rétropéritonéal traumatique du muscle iliaque est une cause rare mais potentiellement sérieuse de neuropathie fémorale compressive. Cas clinique: Nous décrivons les aspects cliniques, radiologiques et thérapeutiques d'un cas d'hématome rétropéritonéal traumatique de la loge iliaque ayant entraîné subséquemment des manifestations de neuropathie fémorale. Discussion: Nous insistons sur le substrat anatomique sous-jacent à la formation d'un hématome avec compression subaiguë du nerf fémoral. Un syndrome compartimental subaigu avec oedème progressif, gonflement et ischémie de la loge iliaque est suggéré comme cause sous-jacente. Une fasciotomie précoce avec ou sans évacuation de l'hématome devrait être considérée dans le but d'obtenir une décompression rapide et ainsi minimiser le risque de lésion nerveuse permanente.

Can. J. Neurol. Sci. 2001; 28: 155-158

Femoral compression neuropathy is a well-recognized entity associated with hemophilia, ${ }^{1-3}$ anticoagulation therapy, ${ }^{4-6}$ cardiac catheterization $^{7,8}$ and major abdomino-pelvic operations. ${ }^{9-11}$ Occasionally, it is a consequence of traumatic iliacus hematoma due to combat $^{11}$ or sporting activities. ${ }^{12,13}$ Typically, patients initially experience sudden onset of severe pain in the inguinal region with associated tenderness in the iliac fossa. Many of them develop various combinations of femoral sensory and motor neuropathy in a delayed fashion. ${ }^{14}$ We report a case of traumatic iliacus hematoma that presented with late onset femoral neuropathy. The pathogenesis of neuropathy and its appropriate management is discussed.

\section{CASE REPORT}

A 15-year-old healthy male sustained a hip extension injury when falling backward on ice while skating. He developed intense pain in the left anterior groin region that continued for about five days. The ultrasound of the groin region was reported as normal. The pain subsequently subsided to be replaced by progressive numbness and weakness in the femoral nerve distribution. There was no history of bleeding diathesis.

Ten days after initial injury, his neurological exam revealed slight weakness of hip flexion (grade 4/5, Medical Research Council), with no contraction of quadriceps (grade 0), normal adduction and absent knee jerk. There was profoundly decreased sensation in the femoral (including saphenous) and lateral femoral cutaneous nerve distributions. A Tinel's sign could be elicited by tapping over the course of these nerves, just above the inguinal ligament. Electrodiagnostic studies were not performed given the short time interval between the onset of neuropathy and clinical presentation.

Computerized tomography (CT) scan revealed a $5 \times 12 \mathrm{~cm}$ iliac fossa hematoma elevating the left iliacus muscle with an ill-defined lucent halo between the lesion and iliacus muscle (Figure).

Exposure of this area through a left retroperitoneal approach revealed stretching of the femoral and lateral femoral cutaneous nerve between the muscle below and tense iliacus fascia above. After iliacus fasciotomy, the sub-iliacus hematoma was evacuated. The underlying iliacus muscle was found to be discoloured and grossly necrotic in appearance. A source of bleeding was not identified.

From the Division of Neurosurgery, University of Toronto (FP) and the Division of Neurosurgery, Department of Surgery, Sunnybrook \& Women's College Health Sciences Centre, University of Toronto (RM), Toronto, ON Canada Received August 23, 2000. Accepted in final form December 5, 2000. Reprint requests to: Rajiv Midha, A-131, Division of Neurosurgery, Sunnybrook \& Women's College Health Sciences Centre, University of Toronto, 2075 Bayview Avenue, Toronto, ON, M4N 3M5 Canada 
Twelve months later, examination revealed normal hip flexion function with significant improvement in quadriceps power (grade 4/5). There was slight residual decreased sensation in the saphenous nerve distribution. Two years postoperatively, the patient has a normal neurological examination.

\section{Discussion}

\section{Clinical}

The association of iliopsoas hematoma and femoral neuropathy has been known since the early part of this century. The hematoma may occur secondary to trauma, including surgical, or spontaneously, especially in patients with abnormal coagulation status. ${ }^{6,12,15,16}$ Although relatively uncommon, it can cause severe permanent disability.

With hemorrhage within the iliacus muscle or subfascial region, the patient initially develops a severe pain in the groin and inguinal region with associated tender globular swelling in the iliac fossa. Occasionally, the hematoma extends into the psoas fascia inferiorly and causes additional fusiform swelling of the psoas compartment, with a palpable groove between these two muscles. ${ }^{17}$ The swelling can also extend into the groin. Patients typically keep their hip flexed, abducted and externally rotated to reduce tension on the iliopsoas muscle. Manifestations of femoral neuropathy will develop later, with its most severe form characterized by weakness and wasting of quadriceps muscle, decreased or absent knee jerk and sensory loss over the anteromedial aspect of the thigh and medial aspect of the lower leg. Variable hip flexion weakness due to iliacus muscle injury or its denervation may also accompany the clinical picture.

Electrodiagnostic investigation with electromyography and nerve conduction studies should be used to establish the femoral nerve functional status and to exclude other neural involvement. Radiographic investigation with CT scan of the iliopsoas compartment is not very specific. However, it can confirm the location and size of the lesion. ${ }^{18}$ A magnetic resonance imaging scan will allow better soft tissue discrimination in cases inadequately imaged by CT, and allow differentiation between hematoma and other lesions.

\section{Anatomical relationship and pathogenesis}

The femoral nerve arises from the dorsal divisions of L2-4 ventral primary rami. It descends through the psoas major toward its inferolateral border, passes between the psoas and iliacus groove deep to the iliacus fascia down to the thigh behind the inguinal ligament. Goodfellow, ${ }^{3}$ using infusion experiments, concluded that the fascial compartment of the iliacus and psoas muscle are separate except for a communication in the thigh below the inguinal ligament. Later, Nobel et al ${ }^{17}$ demonstrated as many as three distinct fascial layers reinforcing the distal portion of the iliacus fascia which offer rigidity to this region and provide the potential for compartment syndrome.

The blood supply to the iliacus muscle is mainly from the iliac branches of the anterior division of the iliolumbar artery. ${ }^{19}$

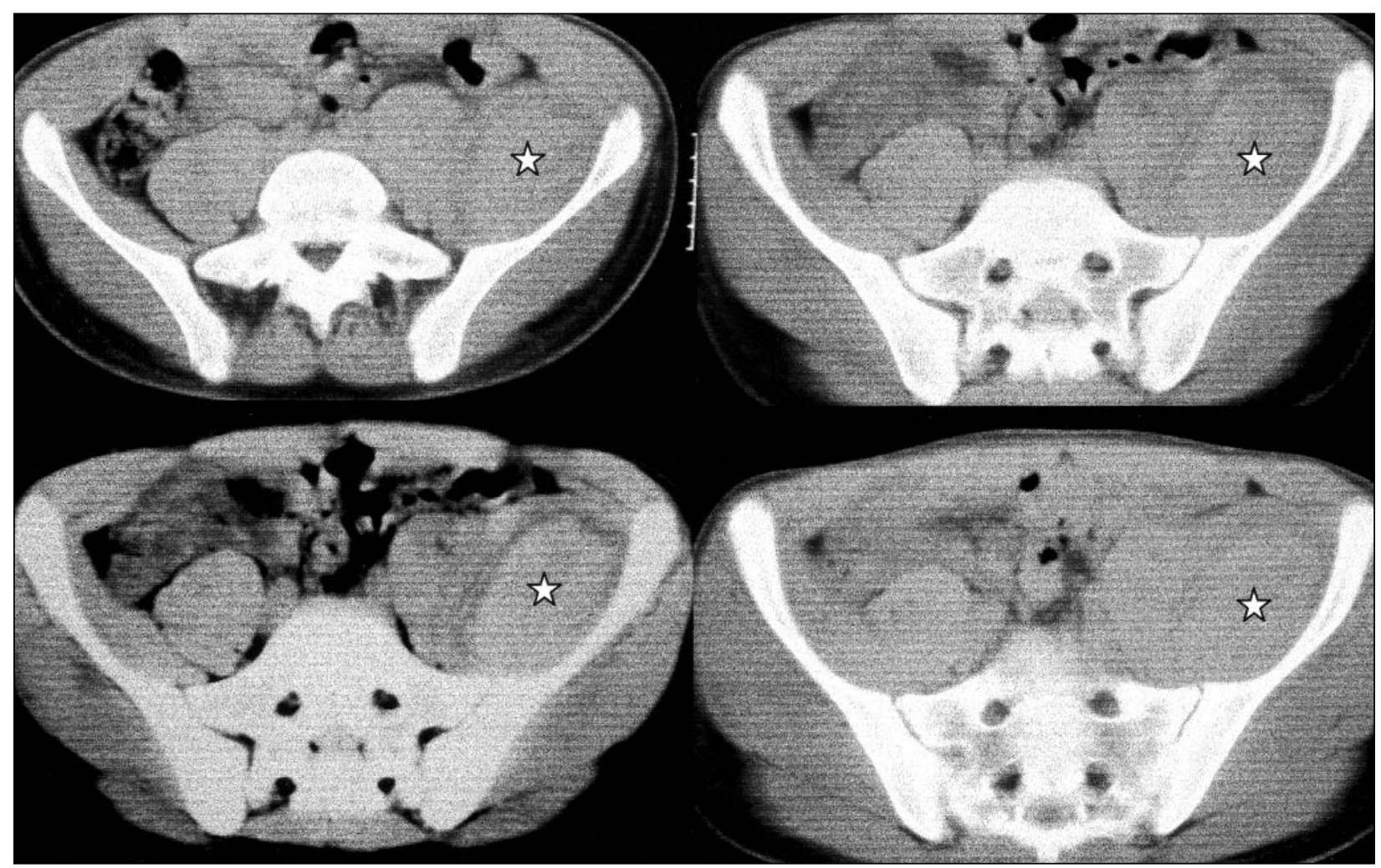

Figure: Computerized tomography scan cuts at the upper pelvic level demonstrate a large soft tissue density mass in the left iliac fossa. The lesion (star) elevates the iliacus muscle away from bone and pushes the psoas muscle medially. At surgery, a huge subacute hematoma was encountered and evacuated. 
The terminal branches of these arteries divide into a superficial division, which runs on the pelvic surface of the iliacus muscle and a deep branch, which passes between the iliacus muscle and periosteum to give rise to a nutrient artery of iliac bone. These branches, mainly the deep ones, are avulsed and torn due to violent contraction and stretching of the iliacus muscle during traumatic injury. This is probably the source of most posttraumatic iliacus hematoma.

The pathogenesis of femoral neuropathy has been related to iliac muscle hematoma. Bleeding into the "iliacus compartment" will subject the femoral nerve to ischemia and compression neuropathy. Our case presented with new onset of neurological deficit about nine days after acute trauma, which presumably initiated the iliacus hematoma. Although several possible sites for hematoma have been described, this case clearly represents submuscular hematoma, possibly due to avulsion of the deep branch of the iliac muscle artery toward the ilium. The frequent delay in neurological presentation after acute inguinal pain may be due to missed diagnosis in the early painful stage of the disease, particularly in bedridden patients. However, the majority of cases likely develop a truly delayed neuropathy from the progressive increase in iliacus compartment pressure with ensuing neuropathy. In our case, initial pain subsiding in about five days, followed by a four-day painless period that merged to painless neuropathy, supports the notion of a progressive compression and /or ischemic neuropathy.

Progressive bleeding may be an additional contributory factor. With increasing pressure in the osseofascial compartment and progressive ischemia of the capillary wall in the muscle with subsequent swelling, interstitial edema and reflex vasospasm in the muscle becomes more important in maintaining the vicious cycle of progressive ischemia and hypertension in the iliacus compartment. This, in turn, could lead to muscle fiber necrosis. Considering the anatomical substrates in the iliac fossa region for development of typical compartment syndrome and previous reports of intraoperative finding of iliacus muscle necrosis associated with neuropathy, ${ }^{3}$ we feel that many patients with this clinical syndrome have developed muscle necrosis at the time of neuropathy. This necrosis will not be functionally significant and, in the context of femoral neuropathy, may go unnoticed. ${ }^{7}$

The femoral nerve is susceptible to compression, especially in the pelvis in subfascial plane, due to its supply by a single nutrient from the iliacus muscle artery. In our case, the radiographic picture demonstrates a significant amount of fluid around the muscle, which suggests a leaky vascular state due to the above mechanism. Lack of direct correlation between the hematoma size and development of the clinical syndrome may be explained by variable fascial compliance in different patients, as well as the rapidity with which hematomas develop. This later factor also has relevance as to the source of bleeding which, in case of low flow venous hemorrhage (e.g., coagulopathy), may be better tolerated.

\section{Management}

Treatment of femoral neuropathy with iliacus hematoma is controversial. For example, many elderly patients with coagulation-induced hematomas are managed expectantly, ${ }^{8}$ while others have undergone surgical decompression. ${ }^{5,6}$ Instead of open surgical procedures, ${ }^{5,6}$ a less invasive percutaneous drainage of the iliopsoas hematoma can also effectively provide decompression of the femoral nerve and allow functional recovery. ${ }^{20}$ Younger patients, particularly those with athletic injuries, are more likely to be managed surgically. ${ }^{13}$ Since good results have been reported with both conservative $\mathrm{e}^{4,8}$ and surgical treatment, ${ }^{6}$ the natural history and factors that influence outcome need to be evaluated. Kuntzer et $\mathrm{al}^{21}$ have noted that irrespective of etiology, approximately $2 / 3$ of patients demonstrate some functional recovery. They further report that the estimate of axon loss of the femoral nerve (based on stimulation of bilateral femoral nerves and recording from the vastus medialis muscle between 10 days to one month from onset of paresis) appears to be the best prognostic factor for recovery. ${ }^{21}$ Although all patients with less than $50 \%$ estimated axonal loss demonstrate improvement and a minority of patients with estimated axonal loss greater than $50 \%$ do so, it is notable that both patients with iliacus hematoma in their study went on to have excellent recovery despite very disparate initial estimates of axonal loss. Also since the patients in the study by Kuntzer et al do not appear to have undergone surgery, it is unclear if surgical decompression may have influenced outcome..$^{10,14,22}$ Given the rarity of the condition, the frequent delay in the diagnosis, variability of clinical deficit at the time of presentation, different etiologies of iliacus hematoma and disparate management offered, any meaningful conclusion from the literature would be difficult for this uncommon entity. In practice, most cases will continue to be managed on an individual basis.

Our case presented with a large hematoma, which was operatively evacuated and has shown progressive recovery. Based on the proposed pathogenesis, we feel that early fasciotomy with, or without, partial hematoma evacuation should be considered in any patient with acute iliacus hematoma unless it is small. This type of approach has been successful in the only large surgical series published to date, in which 23 of 29 cases were treated in this manner. ${ }^{5}$ Even cases where the hematoma is small should be vigilantly observed, for at least a week after the insult, for any symptoms or signs suggestive of compressive neuropathy, including severe progressive pain or early neurological symptoms. Serial CT scan may also be useful in borderline cases. Patients with progressive increase of hematoma size and advanced femoral neuropathy may have lost their optimal time for decompressive operation due to established ischemia. The role of physiotherapy in the acute phase of the conservatively treated group remained questionable due to its potential aggravation of bleeding and/or swelling in the iliacus sheath. If this line of therapy is selected, vigilant neurological follow-up with associated serial imaging is warranted.

\section{ACKNOWLEDGEMENT:}

The authors thank Catherine A Munro for assistance with the manuscript and figures.

\section{REFERENCES}

1. Stewart JD. Compression and entrapment neuropathies. In: Dyck PJ, Thomas PK, eds. Peripheral Neuropathy. Vol. 2, 3rd Ed. Philadelphia: W.B. Saunders Company, 1993; 961-979.

2. Calverley JR, Mulder DW. Femoral neuropathy. Neurology 1960; 10: $963-976$.

3. Goodfellow J. Iliacus haematoma. A common complication of 
haemophilia. J Bone Joint Surg Br 1967; 49: 748-756.

4. Sasson Z. Spontaneous iliopsoas hematoma in patients with unstable coronary syndromes receiving intravenous heparin in therapeutic doses. Can J Cardiol 1996; 12: 490-494.

5. Guivarc'h M. Hematomas of the iliac psoas: 29 cases. J Chir-Paris 1997; 134: 382-389.

6. Young MR, Norris JW. Femoral neuropathy during anticoagulant therapy. Neurology 1976; 26: 1173-1175.

7. Kent KC, Moscucci M. Retroperitoneal hematoma after cardiac catheterization: prevalence, risk factors, and optimal management. J Vasc Surg 1994; 20: 905-910.

8. Kent KC, Moscucci M. Neuropathy after cardiac catheterization: incidence, clinical patterns, and long-term outcome. J Vasc Surg 1994; 19: 1008-1013.

9. Chen SS, Lin ATL, Chen KK, Chang LS. Femoral neuropathy after pelvic surgery. Urology 1995; 46: 575-576.

10. Kim DH, Kline DG. Surgical outcome for intra- and extrapelvic femoral nerve lesions. J Neurosurg 1995; 83: 783-790.

11. Rakolta GG, Omer GE. Combat-sustained femoral nerve injuries. Surg Gynecol Obstet 1969; 128: 813-817.

12. Kumar S. Posttraumatic hematoma of iliacus muscle with paralysis of the femoral nerve. J Orthop Trauma 1992; 6: 110-112.

13. Giuliani G, Poppi M, Acciarri N, Forti A. CT scan and surgical treatment of traumatic iliacus hematoma with femoral neuropathy: case report. J Trauma 1990; 30: 229-231.
14. Warfel BS, Marini SG, Lachmann EA, Nagler W. Delayed femoral nerve palsy following femoral vessel catheterization. Arch Phys Med Rehabil 1993; 74: 1211-1215.

15. Papastefanou SL, Stevens K, Mulholland RC. Femoral nerve palsy: an unusual complication of anterior lumbar interbody fusion. Spine 1994; 19: 2842-2844.

16. Hudson AR, Hunter GA, Waddell JP. Iatrogenic femoral nerve injuries. Can J Surg 1979; 22: 62-66.

17. Nobel W, Marks SC, Kubik S. The anatomical basis for femoral nerve palsy following iliacus hematoma. J Neurosurg 1990; 52: 533-540.

18. Lenchik L. CT of the iliopsoas compartment: value in differentiating tumor, abscess, and hematoma. AJR Am J Roentgenol 1994; 162: 83-86.

19. Salmon M. Arteries of the muscles of the extremities and the trunk, 1st ed. St. Louis, Mo.: Quality Medical Publishing Inc, 1994:138142.

20. Holscher RS, Leyten FSS, Oudenhoven LFIJ, Puylaert JBCM. Percutaneous decompression of an iliopsoas hematoma. Abdom Imaging 1997; 22: 114-116.

21. Kuntzer T, Van Melle G, Regli F. Clinical and prognostic features in unilateral femoral neuropathies. Muscle Nerve 1997; 20: 205211.

22. Osgaard O, Husby J. Femoral nerve repair with nerve autografts. J Neurosurg 1977; 47: 751-754. 